\title{
A SINGLE BASELINE VLBI SURVEY OF SOUTHERN PEAKED SPECTRUM SOURCES
}

\author{
E.A. KING ${ }^{1}$, P.M. MCCULLOCH ${ }^{2}$, D.L. JAUNCEY ${ }^{1}$, \\ J.E. REYNOLDS ${ }^{1}$, R.A. PRESTON ${ }^{3}$, D.L. MEIER ${ }^{3}$, D.W. MURPHY ${ }^{3}$, \\ A.K. TZIOUMIS ${ }^{1}$, J.E.J. LOVELL ${ }^{2}$, T.D. VAN OMMEN ${ }^{3}$ D.L. JONES ${ }^{3}$ \\ ${ }^{1}$ ATNF, CSIRO, Epping, NSW, Australia \\ 2 University of Tasmania - Hobart, Tas., Australia \\ 3 Jet Propulsion Laboratory, Pasadena, CA, USA.
}

A sample of 38 southern peaked-spectrum radio sources from the Parkes Catalogue have been observed using single-baseline VLBI. Thirty three objects were successfully detected on baselines of $>30 M \lambda$ at $2.3 \mathrm{GHz}$. For 21 of these sources, the flux density in the compact components contributes more than half the total flux density of the radio emission. Twenty sources showed structure more complex than a point-source.

The compact morphology of each source was determined by fitting simple image-plane components to the visibility data. This procedure used a toolbox of simple components and was controlled by a stringent set of rules intended to minimize the possibility of introducing false structure into the models. A detailed comparison with the full images from multi-baseline VLBI observations of a subset of the sources has confirmed the general validity of the resulting models. In particular, this comparison shows that single baseline VLBI observations of $\sim 6-8 \mathrm{~h}$ duration are capable of providing reliable estimates of component separations and relative position angles, as well as position angles and lower bounds on the flux densities of individual components. However, actual flux densities and component sizes can not normally be measured reliably with only a single baseline.

A companion flux density monitoring survey conducted over a threeyear period with the University of Tasmania's $26 \mathrm{~m}$ antenna at both 2.3 and $8.4 \mathrm{GHz}$ showed that the incidence of variability in these objects is systematically low, despite the significant contribution made by compact components. Clearly, despite the morphological similarities, the compact structure in these objects is different from that seen in the more variable flat-spectrum sources. 

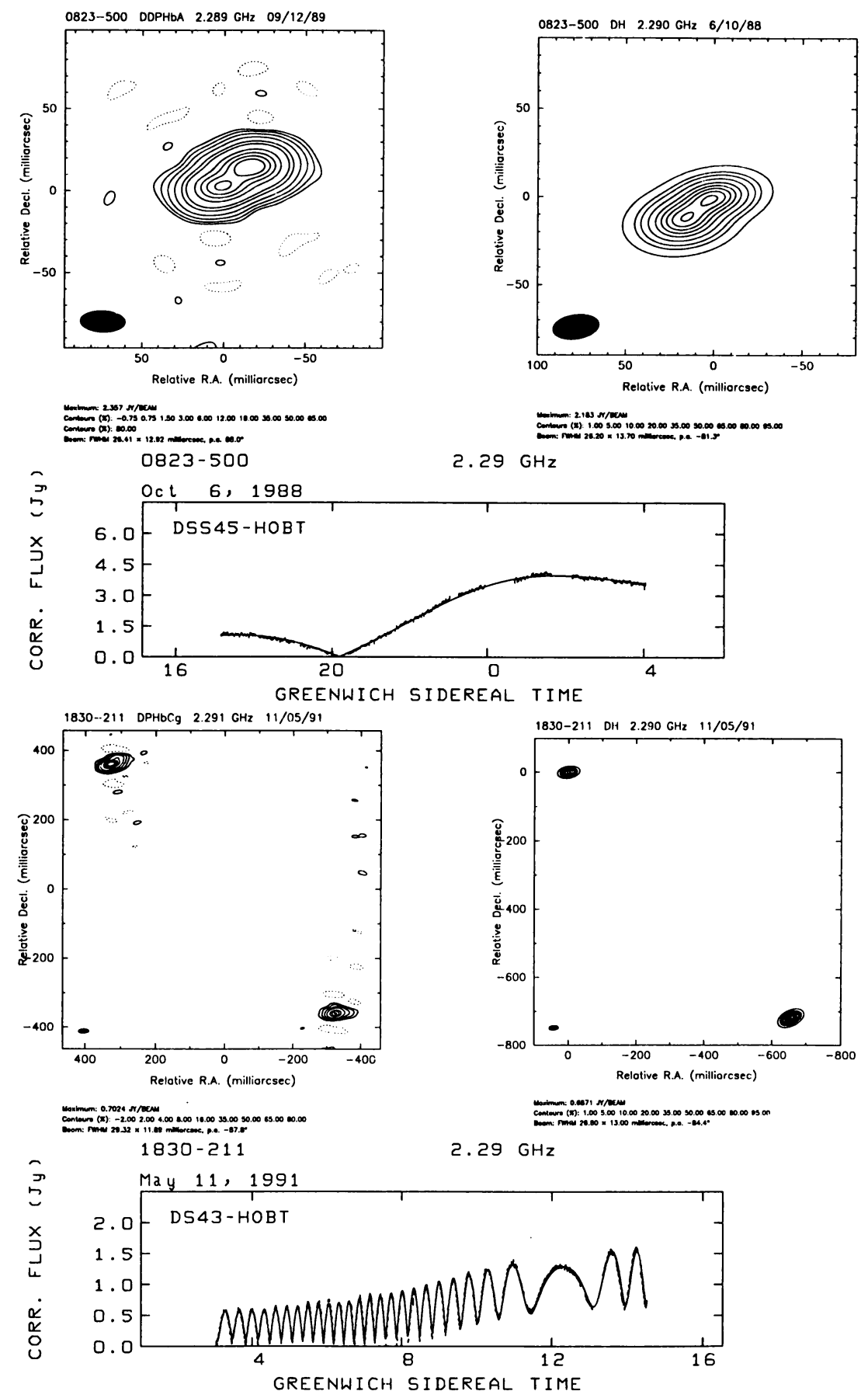

Figure 1. Images, models and modelfits for PKS0823-500 \& PKS1830-211. For each object, the multi-baseline VLBI image is on the left, the single-baseline model on the right. The solid line in the lower plots indicates the model visibility. 